\title{
Introduction to Knowledge Innovation and Entrepreneurial Systems Track
}

\author{
Murray Jennex \\ San Diego State University \\ mjennex@mail.sdsu.edu
}

\author{
Dave Croasdell \\ University of Nevada, Reno \\ davec@unr.edu
}

The knowledge-based view emphasizes the importance of knowledge for organizations to retain their competitive advantage. The success of knowledge sharing practices is vital because it results in shared intellectual capital. Knowledge sharing success lies in the employees' capability to share they know and how they know it. Knowledge systems support sharing practices through their ability to identify, capture, organize, store and disseminate ideas, practices and processes that can impact organizational performance. To the extent such systems can be innovative in their approach to managing organizational resources enables strategies that can foster competitive advantage. Entrepreneurial practices pursue and embed innovation. The Knowledge Innovation and Entrepreneurial Systems track presents research at the intersection of innovative knowledge ideas and practices that enable positive change in organizations. Manuscripts explore systems developed to foster creativity and innovation while facilitating collaboration via knowledge capture, storage and transfer. Evolving infrastructures such as the Internet of Things, the Location of Things and the sharing economy are creating invaluable information assets that, if properly utilized, can deliver positive impacts to organizational performance.

Twelve mini-tracks attracted paper submissions for the KIES research track. The track chairs, mini-track chairs and HICSS administrators seek to preserve and grow the strong community of scholars. We appreciate the efforts of all our colleagues who submitted and/or reviewed papers - as always, the ideas, insights and comments shared through your work are invaluable. Thank you!

AI, Machine Learning, IoT, and Analytics: Exploring the Implications for Knowledge Management and Innovation explores the implications of the deployment and application of AI, Machine Learning, IOT \& Analytics on knowledge management and creation.

Augmenting Human Intelligence: Artificially, Socially, and Ethically presents a study that integrates visual analytics, natural language processing, and deep learning models to augment human intelligence.
Crowd Science focuses on the study of the organizational use of IT-mediated Crowds as a phenomenon to enhance knowledge work.

Culture, International Business, and Knowledge Systems explores the impact and role of culture on managing knowledge and designing and implementing knowledge systems within groups and organizations

Innovation and Entrepreneurship Theory and Practice presents research cases on the role that integration of KM principles and systems may play in facilitating entrepreneurial enterprises.

Intentional Forgetting in Organizations and Information Systems addresses the forgetting paradigm in organizations and information systems. stimulates the discussion in how far and by which means organizations and software systems can and should forget.

Knowledge and Innovation Management for Digitalization and Complexity explores how technology developers need to identify key uncertainties of the innovation during the early phases of the technology's development.

Knowledge Flows, Transfer, Sharing, and Exchange presents manuscripts that examine the nature and role of knowledge flows among entities. Technical, managerial, behavioral, organizational, \& economic perspectives are explored.

Knowledge Management and Information Security investigates issues related to security and protection of intellectual assets and explore how organizations can use security measures to protect their KM practices.

Knowledge Value, Success, and Performance Measurements explores strategies, methodologies, and stories related to performance measurements that can be used to assess various aspects of and success of KM.

Organizational Learning examines KM and organizational learning practices to develop and maximize organizational potential by exploring gaps between learning within organizations and their ability to create, acquire, transfer and manage knowledge.

Reports from the Field considers KM practices by providing a "bridge" between the KM systems practitioner community and the scholars that build theory and conduct research in that domain. 